\title{
Article
}

\section{Reply to Lewin and O'Driscoll: Comment on: "Monitoring of Post-match Fatigue in Professional Soccer: Welcome to the Real World"}

Carling, Christopher, Lacome, M., McCall, A., Dupont, G., Le Gall, F., Simpson, B., and Buchheit, M.

Available at https://clok.uclan.ac.uk/25563/

Carling, Christopher orcid iconORCID: 0000-0002-7456-3493, Lacome, M., McCall, A., Dupont, G., Le Gall, F., Simpson, B., and Buchheit, M. (2019) Reply to Lewin and O'Driscoll: Comment on: "Monitoring of Post-match Fatigue in Professional Soccer: Welcome to the Real World". Sports Medicine . ISSN 0112-1642

It is advisable to refer to the publisher's version if you intend to cite from the work. http://dx.doi.org/10.1007/540279-018-01049-7

For more information about UCLan's research in this area go to http://www.uclan.ac.uk/researchgroups/ and search for < name of research Group>.

For information about Research generally at UCLan please go to http://www.uclan.ac.uk/research/

All outputs in CLoK are protected by Intellectual Property Rights law, including Copyright law. Copyright, IPR and Moral Rights for the works on this site are retained by the individual authors and/or other copyright owners. Terms and conditions for use of this material are defined in the policies page. 


\section{Authors response to Comment on: "Monitoring of Post-match Fatigue in Professional Soccer: Welcome to the Real World'}

Running head: Post-match fatigue in soccer

Authors: ${ }^{1}$ Christopher Carling, ${ }^{2}$ Mathieu Lacome, ${ }^{3,4}$ Alan McCall, ${ }^{5}$ Gregory Dupont, ${ }^{6}$ Franck Le Gall, ${ }^{2}$ Ben Simpson, ${ }^{2}$ Martin Buchheit

Institutions:

${ }^{1}$ Institute of Coaching and Performance, University of Central Lancashire, Preston, UK.

${ }^{2}$ Performance Department, Paris Saint-Germain Football Club, Saint-Germain-en-Laye, France.

${ }^{3}$ Faculty of Health, Life \& Social Sciences, Research Department for Sports and Exercise Science, Edinburgh Napier University, UK;

${ }^{4}$ Arsenal Football Club, Medical Department, London, UK.

${ }^{5}$ The Football Exchange, Research Institute for Sport and Exercise Sciences, Liverpool John Moores University, Liverpool, UK.

${ }^{6}$ Federation Française de Football, Paris, France.

Correspondence:

Christopher Carling, Institute of Coaching and Performance, University of Central Lancashire, Preston, UK.

Phone: +44 (0)1772 891998

Email: ccarling@uclan.ac.uk

ORCID iD: 0000-0002-7456-3493 


\section{Authors response to Comment on: "Monitoring of Post-match Fatigue in Professional Soccer: Welcome to the Real World"}

We would like to thank Lewin \& O'Driscoll (the authors) for their commentary ${ }^{1}$ on our recently published opinion paper $^{2}$ regarding post-match fatigue monitoring (PMF) in elite soccer. We will address in turn what we feel are their main points.

The authors state that the take-home message of our paper errs towards the negative and that PMF monitoring is probably too difficult and not worth introducing in a practical setting. We feel this was not the case and it was certainly not our intention as all the contributing authors to some extent utilize (or have utilized) PMF monitoring in professional club and/or national team soccer environments. However, through a critical in-depth review of the related literature and based on our own experiences as well as exchanges with peers in elite settings, we stand by two of our key summary points: a) owing to a lack of scientific (and anecdotal we may add) evidence, uncertainty still exists around the real-world impact of current research regarding PMF monitoring and its usefulness in informing readiness to play in professional standard soccer players, b) practitioners (e.g., coaching, sports science, medical) must collectively carefully weigh up the need and cost-benefit for PMF monitoring accounting for factors such as logistical burden, coach buy-in, player compliance, exposure time and external workload output in competition, functional relevance of information, a lack of evidence showing that incomplete recovery negatively influences ensuing performance...), and requirements should be determined on a case-by-case basis.

Regarding coach buy-in and player compliance to PMF monitoring, the authors discuss how these factors have successfully evolved at Arsenal FC. This achievement is only to be congratulated and undoubtedly linked to a high level of stability (coaching and medical staff notably) over many years and a club manager who was at the forefront of the upcoming sports science era having already introduced a more systematic approach to preparing players in the late 1990s. The PMF research monitoring programme conducted by Thorpe and colleagues $^{3}$ at Manchester Utd FC is in our opinion another example of good practice and was no doubt aided by strong levels of club stability and recognition and buy-in by the club's coaching staff at that time. We share the same opinion as Lewin \& O'Driscoll in that the way forward is through coach and player education (and we may add upskilling of the former). 'High performance' education in academy players from an early age is necessary while new senior squad signings should encounter PMF monitoring as part of their habitual match/training routine. Unfortunately, the reality is that in many contemporary professional soccer clubs, high levels of player compliance and coach buy-in are not the case, especially outside the English game. Many top players simply cannot be bothered with monitoring and need to be provided with a very good reason. Elite soccer is results-based and coaching staff frequently come and go thereby affecting the continuity of any sports science processes. Indeed, first-team managers are rarely in place for $>2$ years and fighting for fatigue monitoring processes and support staff might be far from their priorities. In an ideal world, processes should remain in place as should support staff, aided through recognition and support by chief stakeholders. In reality however, how often is this the case?

The authors discuss a simple example we made relating to how practitioners weigh up the cost-versus-benefit between allowing a player an additional half/full days rest or missing a key tactical session, for example, to recover a substantial $6.6 \%$ decrease in power derived from a countermovement-jump 24-h following match-play? They state that in their experience, they have never made a recommendation or decision based on the results of only 
one monitoring tool, as in this example. We agree that no objective decision (and we would never try to make one) on player fatigue and readiness to play can be made using the results derived from a single assessment. However, the question arises; if multiple assessments are employed and discrepancies occur across results (e.g, medium decline in countermovement jump performance, large increase in creatine kinase concentration, trivial decline in subjective perceptions of wellbeing) then what would be the authors' decision tree regarding a player's readiness to play status?

The authors also implied that we state recovery strategies should be prioritised over the collection of information on player fatigue. We actually stated that in our experience recovery processes are prioritised by clubs at elite levels over the collection of information on fatigue. The authors subsequently argue why not try to collect information that could make the recovery strategy even more effective? Indeed, they state their work occasionally influences coaching decisions such as adaptations in training load and/or recovery protocols. It would have been extremely useful if they (and other researchers/practitioners generally) could provide real-world examples of PMF monitoring in elite settings. For example, choice of tools and scientific rationale for usage, timing of testing, datasets generated, the magnitude of changes in individual and collective fatigue status, how these changes are meaningfully interpreted notably in relation to current context and in what way the information is subsequently used to impact practice (recovery modalities prescribed, adjusted workload, selection to play or not) and, finally, in an ideal world, evidence of effectiveness.

Finally, the authors discuss how they have experimented with different methods (e.g., technology, software \& staffing) to help ease logistical burden while the progressive gathering of data has enabled them to refine collection and analysis. If any monitoring tool failed to show sufficient sensitivity to detect changes, this has been removed. Yet they also state that a 20-30-minute slot pre-training is required for monitoring. Whilst the authors did not provide information on the specific tests and protocols they employ, this logistical burden still leans in the direction of our recommendation to move towards more practical and ecologically sound assessments for gaining insights into the fatigue status of athletes, without of course questioning the current value of the sports scientist/medical practitioner. We feel there is a clear need to conduct more 'invisible' monitoring, for example using non timeconsuming day-to-day training drills as described in recent works ${ }^{4,5,6}$.

\section{References:}

1. Lewin, C, O'Driscoll, G. Comment on: "Monitoring of Post-match Fatigue in Professional Soccer: Welcome to the Real World' Sports Med. In press.

2. Carling C, Lacome M, McCall A, Dupont G, Le Gall F, Simpson B, et al. Monitoring of post-match fatigue in professional soccer: welcome to the real world. Sports Med. 2018;48:2695-2702.

3. Thorpe RT. Monitoring player fatigue status in the English Premier League. Br J Sports Med. 2018;52:1473-1474.

4. Buchheit M, Simpson BM. Player-Tracking Technology: Half-full or half-empty glass? Int J Sports Physiol Perform. 2017;12(Suppl 2):S235-S241.

5. Rowell AE, Aughey RJ, Clubb J, Cormack SJ. A Standardized Small Sided Game Can Be Used to Monitor Neuromuscular Fatigue in Professional A-League Football Players. Front Physiol. 2018;9:1011. 
6. Delaney, J. The paradox of "invisible" monitoring: The less you do, the more you do! 2018. https://hiitscience.com/the-paradox-of-invisible-monitoring-the-less-you-do-the-moreyou-do/. Accessed 11/12/2018.

\section{Compliance with Ethical Standards}

\section{Funding}

No sources of funding were used to assist in the preparation of this letter.

\section{Conflicts of Interest}

Declare that they have no conflicts of interest relevant to the content of this letter. 